\title{
Effect of maternal age on the outcomes of in vitro fertilization and embryo transfer (IVF-ET)
}

\author{
YAN JunHao ${ }^{\dagger}$, WU KeLiang ${ }^{\dagger}$, TANG Rong, DING LingLing \& CHEN Zi-Jiang * \\ Centre for Reproductive Medicine, Provincial Hospital affiliated to Shandong University, Jinan 250021, China
}

Received April 23, 2012; accepted June 22, 2012

\begin{abstract}
This is a retrospective, observational study to evaluate the effect of maternal age on the outcomes of in vitro fertilization and embryo transfer (IVF-ET). 11830 IVF-ET cycles from 10268 women were included. Four groups of different maternal age periods were compared. The groups were 21-30 years old group (4549 cycles), 31-35 years old group (4424 cycles), 36-40 years old group ( 2429 cycles), and over 40 years old group ( 428 cycles). The mean starting dose of Gn and mean total dose of Gn in each cycle were significantly higher $(P<0.01)$, while the mean retrieved oocyte number was significantly lower $(P<0.01)$ in groups of higher maternal age period than those in each of the lower groups. The biochemical pregnancy rate and the clinical pregnancy rate were significantly lower $(P<0.01)$, while the miscarriage rate was significantly higher $(P<0.01)$ in groups of higher maternal age period than those in the lower groups. No difference was found in two-pronuclear zygotes (2PN) rate and good quality embryo rate among different groups. Birth defect rate was also comparable in the born babies in different groups. In the group with patients' age over 40 years old, the pregnancy rate was $26.87 \%$, the clinical pregnancy rate was $19.39 \%$, while the miscarriage rate after clinical pregnancy was $36.14 \%$. To draw the conclusion, patients with higher maternal age had worse IVF outcomes. In women of fertile age, patients between 20 and 30 years old have the best IVF outcomes. Patients over 40 years old have poor IVF outcome and high miscarriage rate, which suggested the necessity of preimplantation genetic screening (PGS).
\end{abstract}

in vitro fertilization, outcome, pregnancy rate, miscarriage rate, birth defect, maternal age

Citation: Yan J H, Wu K L, Tang R, et al. Effect of maternal age on the outcomes of in vitro fertilization and embryo transfer (IVF-ET). Sci China Life Sci, 2012, 55: 694-698, doi: 10.1007/s11427-012-4357-0

Assisted reproductive technologies have helped many families to have their healthy offspring during the past decades. In vitro fertilization and embryos transfer (IVF-ET) has become increasingly popular and the pregnancy rate was improved by the development of the technology [1,2]. Many women in advanced age want to have babies through IVF. However, it has been reported that one of the main limiting factors to fertility and reproductive outcomes was female's age $[3,4]$.

Advanced maternal age may cause the aging of oocyte

$\dagger$ Contributed equally to this work

*Corresponding author (email: chenzijiang@ hotmail.com) which will result in abnormal fertilization and development, such as polyspermy, division arrest, implantation failure and miscarriage. In addition, it has been demonstrated that the increasing aneuploidy rates in human oocytes and embryos are observed relative to advanced maternal age [5].

Women over 38 years old had poor IVF outcomes, especially in women over 40 years old [6]. Then which age period is the best period for pregnancy? Are IVF outcomes worse in women between 30 and 35 years old than those in women under 30 years old? Not many papers mentioned this.

In this study, we examined the difference of IVF outcomes among groups of different maternal age periods 
(21-30, 31-35, 36-40, and over 40) and found that maternal age has obvious influence on IVF.

\section{Materials and methods}

All women undergoing IVF in the Centre for Reproductive Medicine, Provincial Hospital affiliated to Shandong University between January 2008 and December 2011 were included in the retrospective study. All of these women had undergone thorough IVF treatment according to established protocols.

Oocyte retrieval was performed $32-36 \mathrm{~h}$ after administration of HCG trigger guiding by standard transvaginal ultrasound and plasma LH level. Collected oocytes were fertilized in vitro and 1-3 good quality embryos were transferred on day 3 or day 5. Progesterone was used for luteal phase support regularly. Biochemical pregnancy was verified by plasma $\beta$-HCG $>20 \mathrm{IU} \mathrm{L}^{-1} 14 \mathrm{~d}$ after embryo transfer. Clinical pregnancy was defined as pregnancy sac found in the uterus using B ultrasound $20 \mathrm{~d}$ after biochemical pregnancy confirmation.

\subsection{Patients}

A total of 11830 IVF-ET cycles from 10268 women were included in this study. The IVF outcomes were analyzed in four different groups sorted by the age period. The groups were 21-30 years old group (4549 cycles), 31-35 years old group (4424 cycles), 36-40 years old group (2429 cycles), and over 40 years old group (428 cycles).

\subsection{IVF outcomes measurement}

Fertilization was determined by the presence of pronuclei 18-20 h after insemination, only the fertilized oocytes with two-pronuclear zygotes (2PN) were classified to normal fertilized group. The embryos derived from $2 \mathrm{PN}$ zygotes were graded as good quality when the number of blastomeres was between 7 and 12; additionally, all of the blastomeres had equal or almost equal size, and the percentage of anucleated fragments was $<25 \%$ on day 3 .

Miscarriage was defined as a spontaneous loss of a clinical pregnancy before 24 weeks of gestation; birth defect was defined as one causing death or serious disability [7].

The mean starting dose of Gonadotropin (Gn), mean total dose of $\mathrm{Gn}$ in each cycle, mean retrieved oocyte number, 2PN zygotes rate (2PN zygotes number/retrieved oocytes), good quality embryo rate (good quality embryo/2PN zygotes), biochemical pregnancy rate (biochemical pregnancy cycles/embryo transfer cycles), clinical pregnancy rate (clinical pregnancy cycles/biochemical pregnancy cycles), miscarriage rate (miscarriage cycles/clinical pregnancy cycles), and birth defect rate were compared among different age groups.

\subsection{Statistical analysis}

Statistical analysis was performed with the use of SPSS 19.0. A $P$-value of $<0.05$ was considered to be statistically significant.

\subsection{Ethics}

This study was a retrospective analysis on the clinical practice outcomes and the data of our study were approved by the Institutional Review Board of Shandong University.

\section{Results}

A total of 11830 IVF-ET cycles were included in this study.

The IVF-ET outcomes were analyzed in four different groups classified by the age periods. The groups were 21-30 years old group (4549 cycles), 31-35 years old group (4424 cycles), 36-40 years old group (2429 cycles), and over 40 years old group (428 cycles).

As shown in Table 1 and Figures 1-4, the mean starting dose of $\mathrm{Gn}$ and mean total dose of $\mathrm{Gn}$ of each cycle were significantly higher $(P<0.01)$ while the number of mean retrieved oocyte was significantly lower $(P<0.01)$ in groups of higher maternal age period than those in each of the lower groups. The biochemical pregnancy rate and the clinical pregnancy rate were significantly lower $(P<0.01)$, while the miscarriage rate was significantly higher $(P<0.01)$ in groups of higher maternal age period than those in each of the lower groups.

No difference was found in 2PN zygotes rate and good quality embryo rate among different groups. Birth defect rate was also comparable in the born babies. In the group with patients' age over 40 years old, the pregnancy rate was $26.87 \%$, the clinical pregnancy rate was $19.39 \%$, while the miscarriage rate after clinical pregnancy was $36.14 \%$ (Table 1, Figure 4).

\section{Discussion}

It is recognized by many reporters that with the increasing of maternal age, the IVF outcomes become increasingly worse [3]. Some IVF centers even limited the last age for IVF as 43 years old [8]. Women with advanced maternal age will have poor ovary response during controlled ovary hyperstimulation $(\mathrm{COH})$, low retrieved oocyte number, lowoocyte fertilization rate, low good quality embryo rate, low embryo implantation rate, low pregancy rate, high miscarriage rate, high preterm delivery rate and high birth defect rate [6,9-12].

We found that first of all, in our study, the fertility significantly dropped in women over 30 years old, which is earlier than some other reports of the late 30s [11]. In our study, 


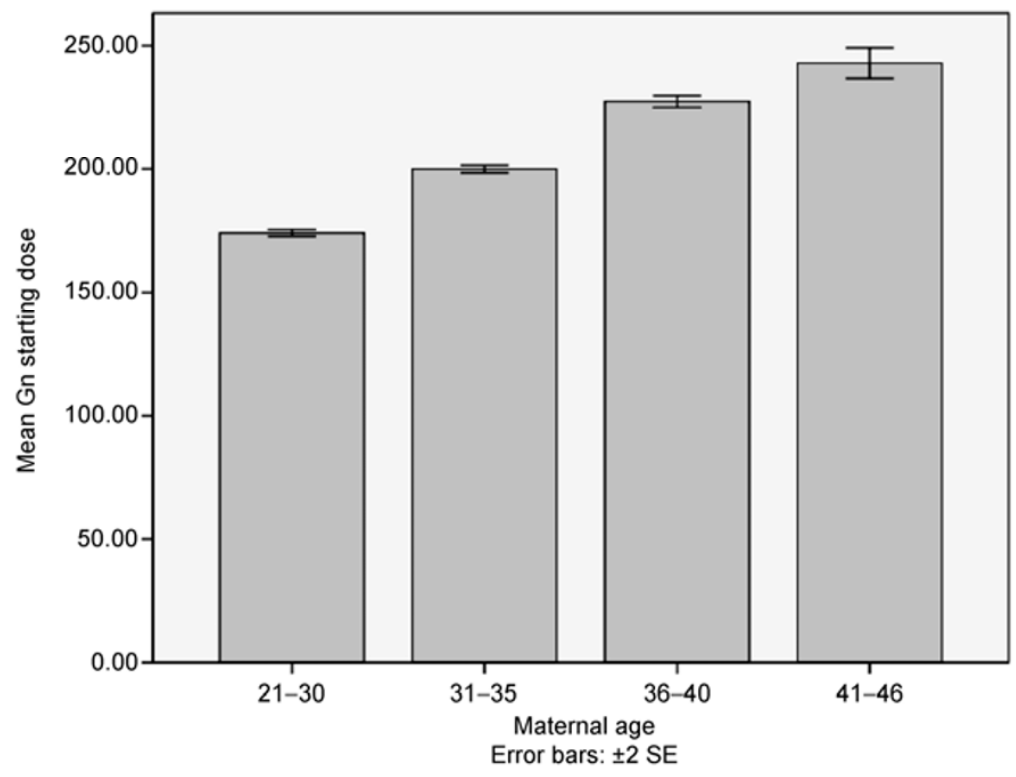

Figure 1 Mean $\mathrm{Gn}$ starting dose (IU L L ${ }^{-1}$ ) in different maternal age period groups.

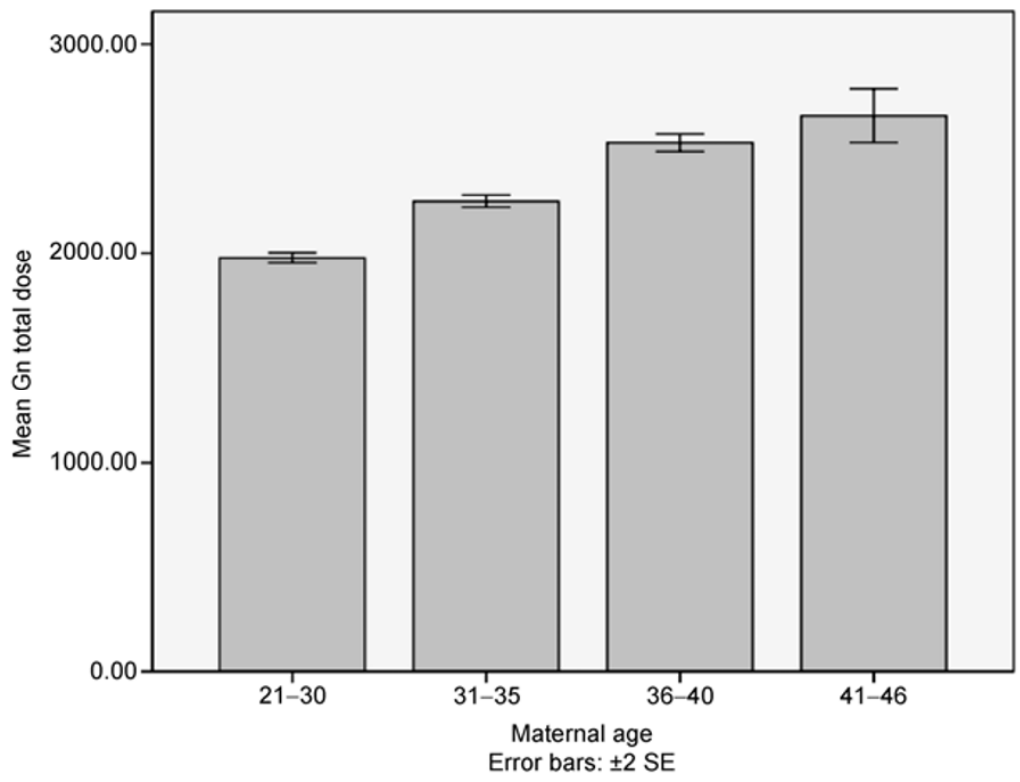

Figure 2 Mean $\mathrm{Gn}$ total dose (IU L $\left.{ }^{-1}\right)$ in different maternal age period groups.

in women of 31-35 age period undergoing IVF, the mean starting dose of $\mathrm{Gn}$ and mean total dose of Gnin each cycle were significantly higher than those in women of 21-30 age period, while the mean retrieved oocyte number was significantly lower. This is even worse in higher age period. This means the ovary poor response starts earlier than we have expected. When a woman is over 30 years old with tubal factor for infertility, IVF should be suggested rather than laparoscopy surgery. It is necessary to suggest women have babies earlier than 30 years old.

Secondly, in our study, the biochemical pregnancy rate and the clinical pregnancy rate were significantly lower, while the miscarriage rate was significantly higher in women with higher maternal age, even in $31-35$ age period. It is reasonable that the inner quality of oocytes and embryos will decrease the implantation rate, hence affecting the pregnancy rate. However, the $2 \mathrm{PN}$ zygotes rate and the good quality embryo rate had no difference among groups in our study, which may be because of the score criteria and selection system in the lab.

Another hot point of IVF outcome is the miscarriage rate after clinical pregnancy. This is an important aspect of IVF achievement rate. Previous studies have suggested an association between the reduced fertility and an increased risk of miscarriage [13-15]. We found in our study over $1 / 3$ patients miscarried after clinical pregnancy in women under- 


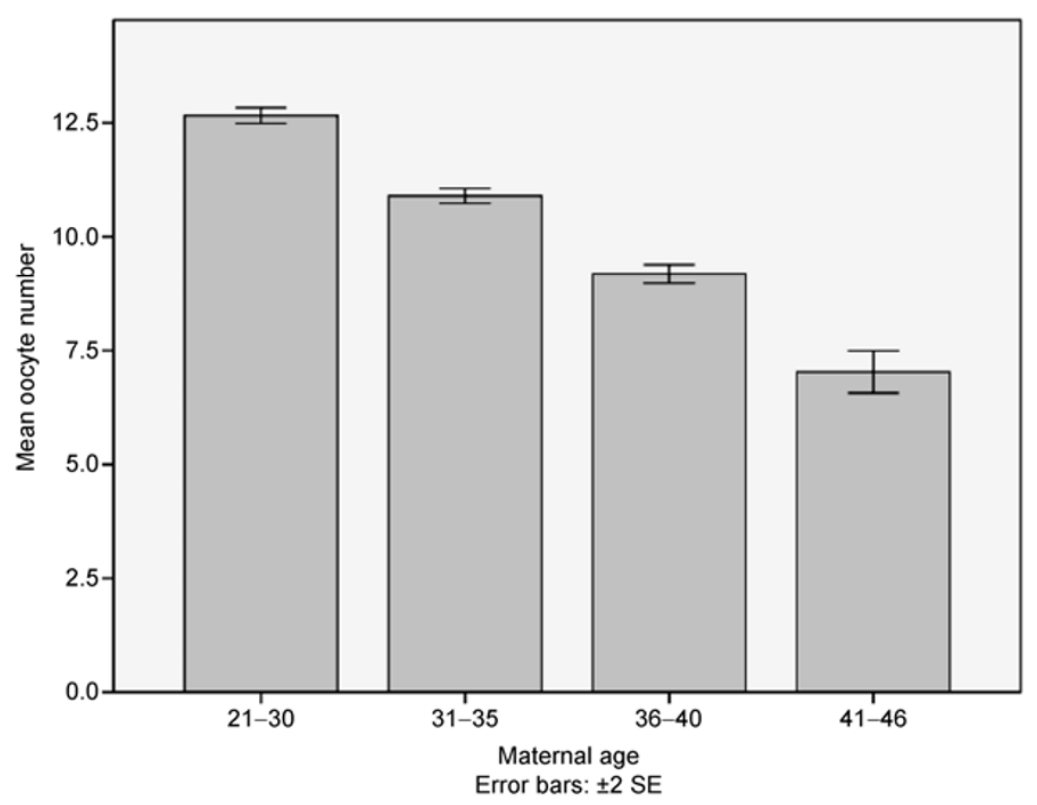

Figure 3 Mean retrieved oocyte number in different maternal age period groups.

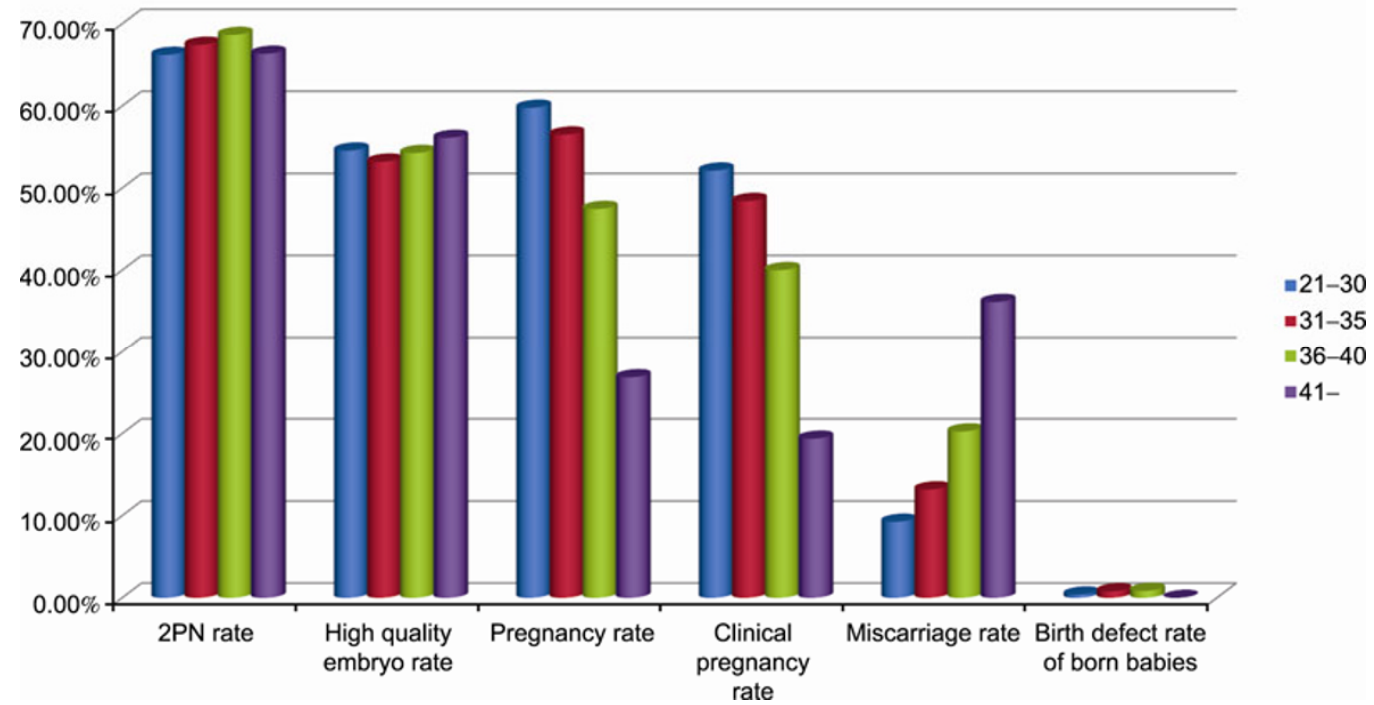

Figure 4 IVF outcomes of different maternal age periods.

going IVF over 40 years old; this rate is $20.27 \%$ in $36-40$ years old group, $13.21 \%$ in $31-35$ years old group and only $9.27 \%$ in $21-30$ years old group. In the latter two groups, the miscarriage rates after diagnosis of clinical pregnancy are comparable to previous reports of natural pregnancy $(10 \%-15 \%)$ [16-19]. It is reported that genetic abnormalities, uterine anatomical defects, antiphospholipid antibodies, environmental factors, advanced maternal age, obesity, endometriosis, etc. may be the possible causes of miscarriage in natural and assisted conceptions [20-24]. In our study, the higher miscarriage rates in patients over 35 years old may mainly result from advanced maternal age and aneuploid embryo since most of the other reasons were excluded before IVF cycle starting.
PGS was encouraged in women over 40 years old [25], though the latest meta-analysis discourage the using of PGS [26], patients selection is an important procedure in PGS. Comparative genomic hybridization $(\mathrm{CGH})$ and array-CGH were widely used now for PGS which could make the result more sensitive and exact [27-30].

Finally, in our study, we found that the birth defect rates have a trend to go up by the increasing of maternal age, but no significant difference was found. It is a pity that some of the pregnancies were still in progress when this paper was drafted, so we could not compare the live birth rate or take home baby rate with the relatively small number of birth.

To draw the conclusion, patients with higher maternal age had worse IVF outcomes. Patients between 20 and 30 
Table 1 IVF outcomes of different maternal age periods ${ }^{\text {a) }}$

\begin{tabular}{|c|c|c|c|c|c|c|c|c|c|c|c|c|c|c|}
\hline $\begin{array}{l}\text { Age } \\
\text { period }\end{array}$ & Cycles & $\begin{array}{l}\text { Mean Gn } \\
\text { starting } \\
\text { dose } \\
\left(\mathrm{IU} \mathrm{L}^{-1}\right) \\
\end{array}$ & $\begin{array}{c}\text { Mean Gn } \\
\text { total dose } \\
\left(\mathrm{IU} \mathrm{L}^{-1}\right)\end{array}$ & $\begin{array}{l}\text { Mean } \\
\text { oocyte } \\
\text { number }\end{array}$ & $\begin{array}{l}2 \mathrm{PN} \\
\text { zygotes } \\
\text { rate }\end{array}$ & $\begin{array}{l}\text { High } \\
\text { quality } \\
\text { embryo } \\
\text { rate } \\
\end{array}$ & $\begin{array}{c}\text { Pregnancy } \\
\text { cycles }\end{array}$ & $\begin{array}{l}\text { Pregnancy } \\
\text { rate }\end{array}$ & $\begin{array}{l}\text { Clinical } \\
\text { pregnancy } \\
\text { cycles }\end{array}$ & $\begin{array}{l}\text { Clinical } \\
\text { pregnancy } \\
\text { rate }\end{array}$ & $\begin{array}{l}\text { Implanta- } \\
\text { tion rate }\end{array}$ & $\begin{array}{l}\text { Misca- } \\
\text { rriage } \\
\text { cycles }\end{array}$ & $\begin{array}{l}\text { Misca- } \\
\text { rriage } \\
\text { rate }\end{array}$ & $\begin{array}{l}\text { Birth defect } \\
\text { rate of born } \\
\text { babies }\end{array}$ \\
\hline $31-35$ & 4424 & $\begin{array}{c}199.94 \pm \\
0.76^{* * *}\end{array}$ & $\begin{array}{c}2249.96 \pm \\
13.87^{* *}\end{array}$ & $\begin{array}{c}10.89 \pm \\
0.08^{* *}\end{array}$ & $67.46 \%{ }^{\#}$ & $53.23 \%$ \# & 2501 & $56.53 \%^{* *}$ & 2143 & $48.44 \%^{* *}$ & $30.75 \%^{* *}$ & 283 & $13.21 \%^{* *}$ & $\begin{array}{c}0.81 \% \\
(9 / 1118)^{\#}\end{array}$ \\
\hline $36-40$ & 2429 & $\begin{array}{c}227.45 \pm \\
1.19^{* *}\end{array}$ & $\begin{array}{c}2528.65 \pm \\
21.26^{* *}\end{array}$ & $\begin{array}{l}9.18 \pm \\
0.10^{* *}\end{array}$ & $68.66 \%{ }^{\#}$ & $54.29 \%$ & 1153 & $47.47 \%^{* *}$ & 972 & $40.02 \%^{* *}$ & $21.22 \%^{* *}$ & 197 & $20.27 \%^{* *}$ & $\begin{array}{c}0.86 \% \\
(4 / 463) \mathrm{s}^{\#}\end{array}$ \\
\hline $41-$ & 428 & $\begin{array}{c}242.93 \pm \\
3.08^{* * *}\end{array}$ & $\begin{array}{c}2659.61 \pm \\
65.02^{* *}\end{array}$ & $\begin{array}{l}7.04 \pm \\
0.23^{* *}\end{array}$ & $66.37 \%{ }^{\#}$ & $56.13 \%{ }^{\#}$ & 115 & $26.87 \%^{* *}$ & 83 & $19.39 \%{ }^{* *}$ & $8.83 \%^{* *}$ & 30 & $36.14 \%{ }^{* *}$ & $\begin{array}{l}0.00 \% \\
(0 / 20)\end{array}$ \\
\hline
\end{tabular}

a) $*$, Some pregnancies are still in progress; $* *, P<0.01$ vs. each of the lower age periods; \#, $P>0.05$ vs. each of the lower age periods.

years old have the best IVF outcomes in women of fertile age. Patients over 40 years old have really poor IVF outcomes and high miscarriage rate, which suggested the necessity of preimplantation genetic screening (PGS).

1 Gnoth C, Maxrath B, Skonieczny T, et al. Final ART success rates: a 10 years survey. Hum Reprod, 2011, 26: 2239-2246

2 de Mouzon J, Goossens V, Bhattacharya S, et al. Assisted reproductive technology in Europe, 2006: results generated from European registers by ESHRE. Hum Reprod, 2010, 25: 1851-1862

3 Balasch J. Ageing and infertility: an overview. Gynecol Endocrinol, 2010, 26: 855-860

4 Tatone C. Oocyte senescence: a firm link to age-related female subfertility. Gynecol Endocrinol, 2008, 24: 59-63

5 Hassold T, Chen N, Funkhouser J, et al. A cytogenetic study of 1000 spontaneous abortions. Ann Hum Genet, 1980, 44: 151-178

6 Thum M Y, Abdalla H I, Taylor D. Relationship between women's age and basal follicle-stimulating hormone levels with aneuploidy risk in in vitro fertilization treatment. Fertil Steril, 2008, 90: 315-321

7 Yan J, Huang G, Sun Y, et al. Birth defects after assisted reproductive technologies in China: analysis of 15,405 offspring in seven centers (2004 to 2008). Fertil Steril, 2011, 95: 458-460

8 Hourvitz A, Machtinger R, Maman E, et al. Assisted reproduction in women over 40 years of age: how old is too old? Reprod Biomed Online, 2009, 19: 599-603

9 Griffiths A, Dyer S M, Lord S J, et al. A cost-effectiveness analysis of in-vitro fertilization by maternal age and number of treatment attempts. Hum Reprod, 2010, 25: 924-931

10 Vincent-Rohfritsch A, Le Ray C, Anselem O, et al. Pregnancy in women aged 43 years or older: Maternal and perinatal risks. J Gynecol Obstet Biol Reprod (Paris), 2012 (in press)

11 Liu K, Case A. Advanced reproductive age and fertility. J Obstet Gynaecol Can, 2011, 33: 1165-1175

12 Sandalinas M, Sadowy S, Alikani M, et al. Developmental ability of chromosomally abnormal human embryos to develop to the blastocyst stage. Hum Reprod, 2001, 16: 1954-1958

13 Jansen R P. Spontaneous abortion incidence in the treatment of infertility. Am J Obstet Gynecol, 1982, 143: 451-473

14 Hakim R B, Gray R H, Zacur H. Infertility and early pregnancy loss. Am J Obstet Gynecol, 1995, 172: 1510-1517

15 Liu H C, Jones G S, Jones H W Jr., et al. Mechanisms and factors of early pregnancy wastage in in vitro fertilization-embryo transfer patients. Fertil Steril, 1988, 50: 95-101

16 Risch H A, Weiss N S, Clarke E A, et al. Risk factors for spontaneous abortion and its recurrence. Am J Epidemiol, 1988, 128: $420-430$

17 Edmonds D K, Lindsay K S, Miller J F, et al. Early embryonic mortality in women. Fertil Steril, 1982, 38: 447-453

18 Miller J F, Williamson E, Glue J, et al. Fetal loss after implantation. A prospective study. Lancet, 1980, 2: 554-556

19 Molo M W, Kelly M, Balos R, et al. Incidence of fetal loss in infertility patients after detection of fetal heart activity with early transvaginal ultrasound. J Reprod Med, 1993, 38: 804-806

20 Nybo Andersen A M, Wohlfahrt J, Christens P, et al. Maternal age and fetal loss: population based register linkage study. BMJ, 2000, 320: 1708-1712

21 Cramer D W, Wise L A. The epidemiology of recurrent pregnancy loss. Semin Reprod Med, 2000, 18: 331-339

22 Fedorcsak P, Dale P O, Storeng R, et al. Impact of overweight and underweight on assisted reproduction treatment. Hum Reprod, 2004, 19: 2523-2528

23 Barnhart K, Dunsmoor-Su R, Coutifaris C. Effect of endometriosis on in vitro fertilization. Fertil Steril, 2002, 77: 1148-1155

24 Garcia-Enguidanos A, Calle M E, Valero J, et al. Risk factors in miscarriage: a review. Eur J Obstet Gynecol Reprod Biol, 2002, 102: $111-119$

25 Milan M, Cobo A C, Rodrigo L, et al. Redefining advanced maternal age as an indication for preimplantation genetic screening. Reprod Biomed Online, 2010, 21: 649-657

26 Mastenbroek S, Twisk M, van der Veen F, et al. Preimplantation genetic screening: a systematic review and meta-analysis of RCTs. Hum Reprod Update, 2011, 17: 454-466

27 Traversa M V, Marshall J, McArthur S, et al. The genetic screening of preimplantation embryos by comparative genomic hybridisation. Reprod Biol, 2011, 11: 51-60

28 Chang L J, Chen S U, Tsai Y Y, et al. An update of preimplantation genetic diagnosis in gene diseases, chromosomal translocation, and aneuploidy screening. Clin Exp Reprod Med, 2011, 38: 126-134

29 Geraedts J, Montag M, Magli M C, et al. Polar body array CGH for prediction of the status of the corresponding oocyte. Part I: clinical results. Hum Reprod, 2011, 26: 3173-3180

30 Gutierrez-Mateo C, Colls P, Sanchez-Garcia J, et al. Validation of microarray comparative genomic hybridization for comprehensive chromosome analysis of embryos. Fertil Steril, 2011, 95: 953-958

Open Access This article is distributed under the terms of the Creative Commons Attribution License which permits any use, distribution, and reproduction in any medium, provided the original author(s) and source are credited. 